\title{
Evaluation of Plasticity Models Using Uniaxial Tensile Test
}

\author{
Aysema Ünlü ${ }^{1} \mathbb{D}$, Emre Esener ${ }^{1^{*}} \mathbb{D}$, Mehmet Fırat ${ }^{2}$ \\ 'Mechanical Engineering Department/ Bilecik Seyh Edebali University, Turkey \\ ${ }^{2}$ Mechanical Engineering Department/ Sakarya University, Turkey
}

\begin{abstract}
In this study, it is aimed to evaluate plasticity model prediction performance for plastic behavior of materials using a uniaxial tensile test. For this purpose, von Mises, Hill-48, Hill-93, Barlat-89 and Hu -2003 plasticity models are studied, and DC04, DP780, 6000 series aluminum alloy are used as materials. Tensile tests are performed with three directions (rolling, diagonal, transverse), and mechanical properties of materials are obtained. In addition, anisotropy coefficients of materials are calculated by uniaxial tensile tests. Validation of plasticity models is performed using obtained material parameters. Yield locus and yield stresses-anisotropy coefficients depends on directions are used in evaluation of plasticity models. As a result of this study, $\mathrm{Hu}-2003$ showed the best modeling performance for all materials.
\end{abstract}

Keywords: Plasticity Modeling, Tensile Test, Anisotropy, Yield Locus

\section{INTORDUCTION}

Mechanical behavior can be defined as a behavior of the materials under loading, and properties that determine this behavior can be named as mechanical properties. Mechanical tests can identify the mechanical behavior of materials, and the tensile test has a wide usage area. Force-stroke curves can be obtained as a result of a uniaxial tensile test than stress-strain curves can be calculated to eliminate the dimension factor. Stress-strain curves represent the behavior of a material under uniaxial tensile load. Yield stress, tensile stress, elasticity modulus, strength coefficient, hardening exponent, anisotropy coefficient, etc. can be calculated from a uniaxial tensile test. These mechanical properties can be used as an input for plasticity models in finite element analyses to determine the plastic behavior of materials. Today, a huge number of plasticity models exist for modeling the plastic behavior of materials [1-4]. Modeling elastic behavior of a material can be performed easily by using a linear Hooke equation, however modeling plastic behavior is more complicated and needs information such as initial yield point, stress-strain relation, hardening behavior that identifies the development of yield stress. [5].

Today, finite element analyses become an effective tool that has a wide usage area in both academy, and industry. Prediction performance of finite element analyses has a great importance, especially in industrial analyses. Prediction accuracy of finite element analyses depends on finite element calculation parameters as element size, element for- mulation, number of integration points, contact condition, symmetry condition, and plasticity models [6-9], besides, plasticity models have a dominating effect on prediction performance of finite element analyses [10-11] so, selection of plasticity model becomes an important part of preprocessing stage in finite element analyses. Determining material plastic behavior capacities of plasticity models depends on the assumptions of these criteria. Plasticity models can be classified basically as isotropic material-isotropic hardening, anisotropic material-isotropic hardening, and anisotropic material-kinematic hardening. In this sense, several advanced plasticity models are presented in recent years [12-15]. However, usage limitations of these plasticity models belong to the number of material parameters needed. Today, plasticity models with 18 parameters [16] and plasticity models with 11 parameters [17] are presented in the literature. Plasticity models with fewer parameters have an advantage by means of calculation time thus, users need less number of mechanical tests and mechanical parameters.

As a summary, an effective selection of plasticity model which affects the prediction accuracy of finite element analyses is a critical stage of engineering calculations, especially in industrial applications. In this study, five different plasticity models are evaluated by uniaxial tensile tests. For this purpose, von Mises, Hill-48, Hill-93, Barlat-89, and Hu-2003 plasticity models are evaluated by uniaxial tensile tests with three directions (rolling, diagonal, transverse) for DC04, DP780, and 6000 series aluminum alloy materials are per- 
formed, and plastic behavior representations of plasticity models are compared.

\section{MATERIAL \& METHOD}

In this study, DC04 mild steel, dual-phase DP780 advanced high strength steel, and 6000 series of aluminum alloy are used as materials for evaluating plasticity models. Firstly, uniaxial tensile tests are performed for obtaining mechanical properties and all materials are tested for three different directions (rolling, diagonal, transverse). In addition, uniaxial tensile tests for determining anisotropy coefficients are performed in the same directions. Then, the plastic behavior of materials is modeled using five different plasticity models. For this purpose, yield loci and the angular variations of the yield stress ratio and anisotropy coefficients are calculated by using these models, and predicted results are compared with the experimental results.

\subsection{Experimental Studies}

All tensile tests are performed with a Shimadzu AG-IC tensile test machine. Tensile samples are manufactured using cutting dies from sheet plates (Fig. 1) according to ASTM-E8 standards [18] (Fig. 2). Test velocity is applied as $25 \mathrm{~mm} / \mathrm{min}$ constant. Non-contacted extensometers are used for displacement measurements. Test samples are shown in Fig. 3.

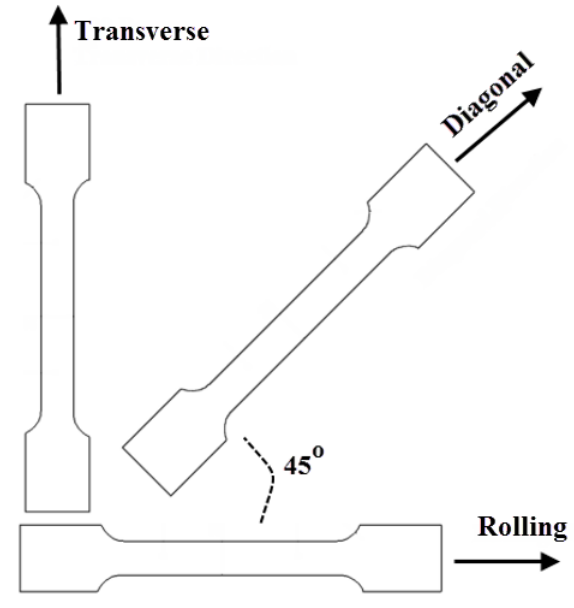

Figure 1. Schematical view of tensile test directions

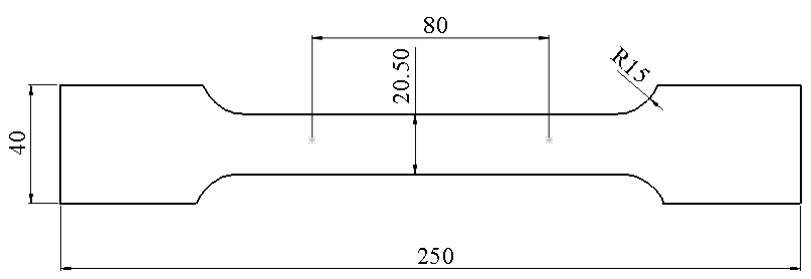

Figure 2. ASTM-E8 tensile test sample

Force-stroke data are converted as stress-strain data and engineering stress-strain curves are obtained for all materials (Fig. 4). Then, experimental flow curves are calculated to determine the plastic behavior of materials. For this purpose, engineering stress-strain curves are converted to true stress-strain curves, after then elastic parts are subtracted from the data. Flow curves of all materials are given in Fig. 5.
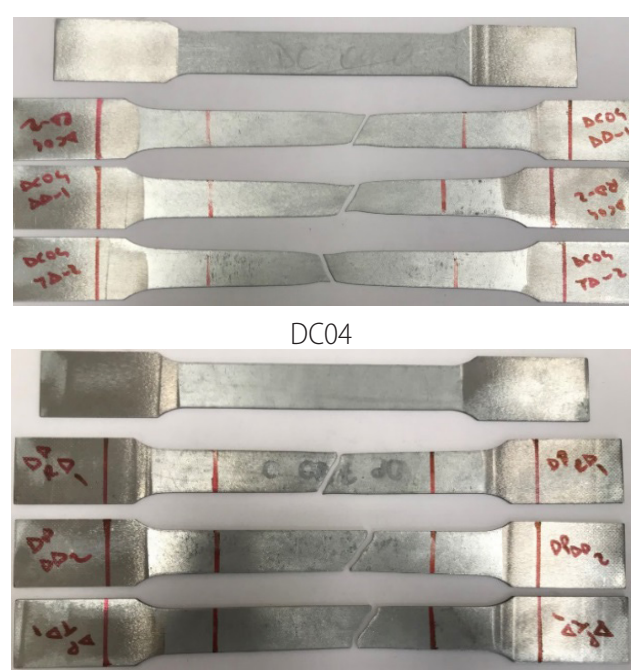

DP780

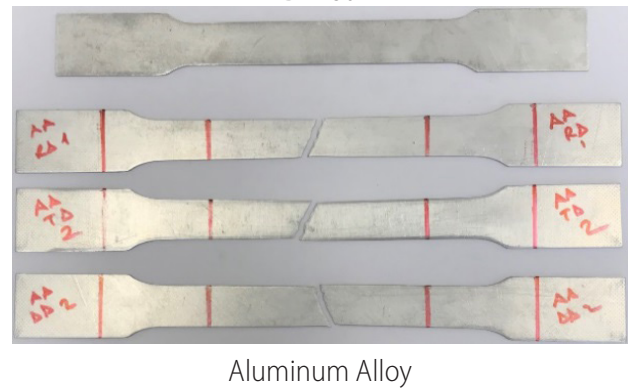

Figure 3. Test samples after uniaxial tensile tests
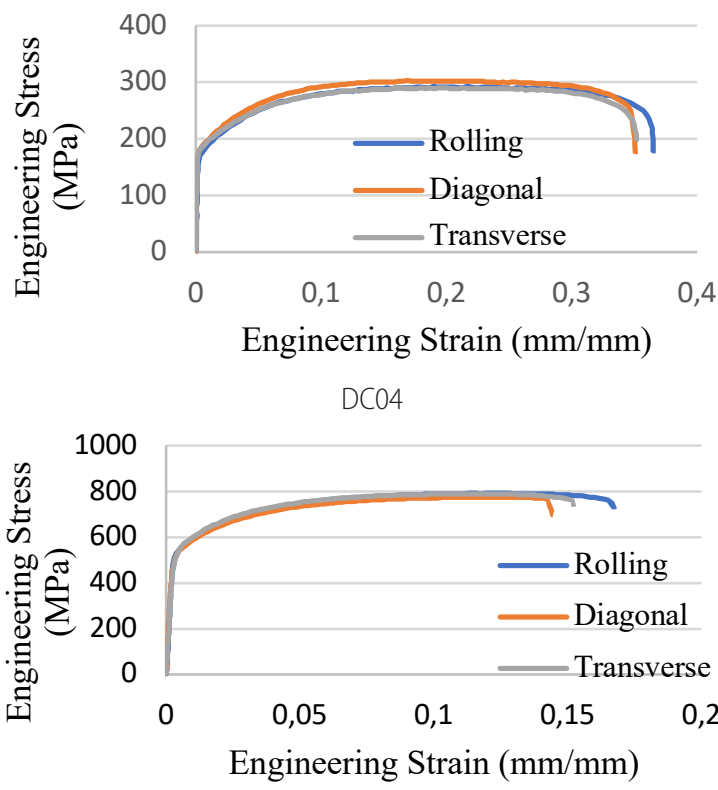

Engineering Strain $(\mathrm{mm} / \mathrm{mm})$

DP780

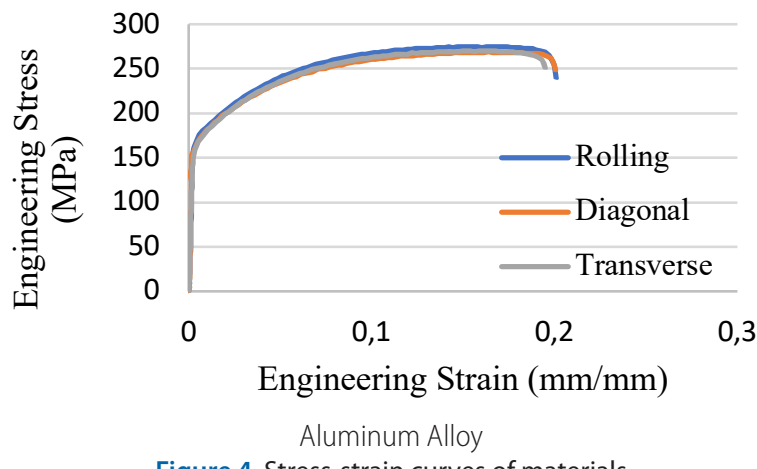

Figure 4. Stress-strain curves of materials 

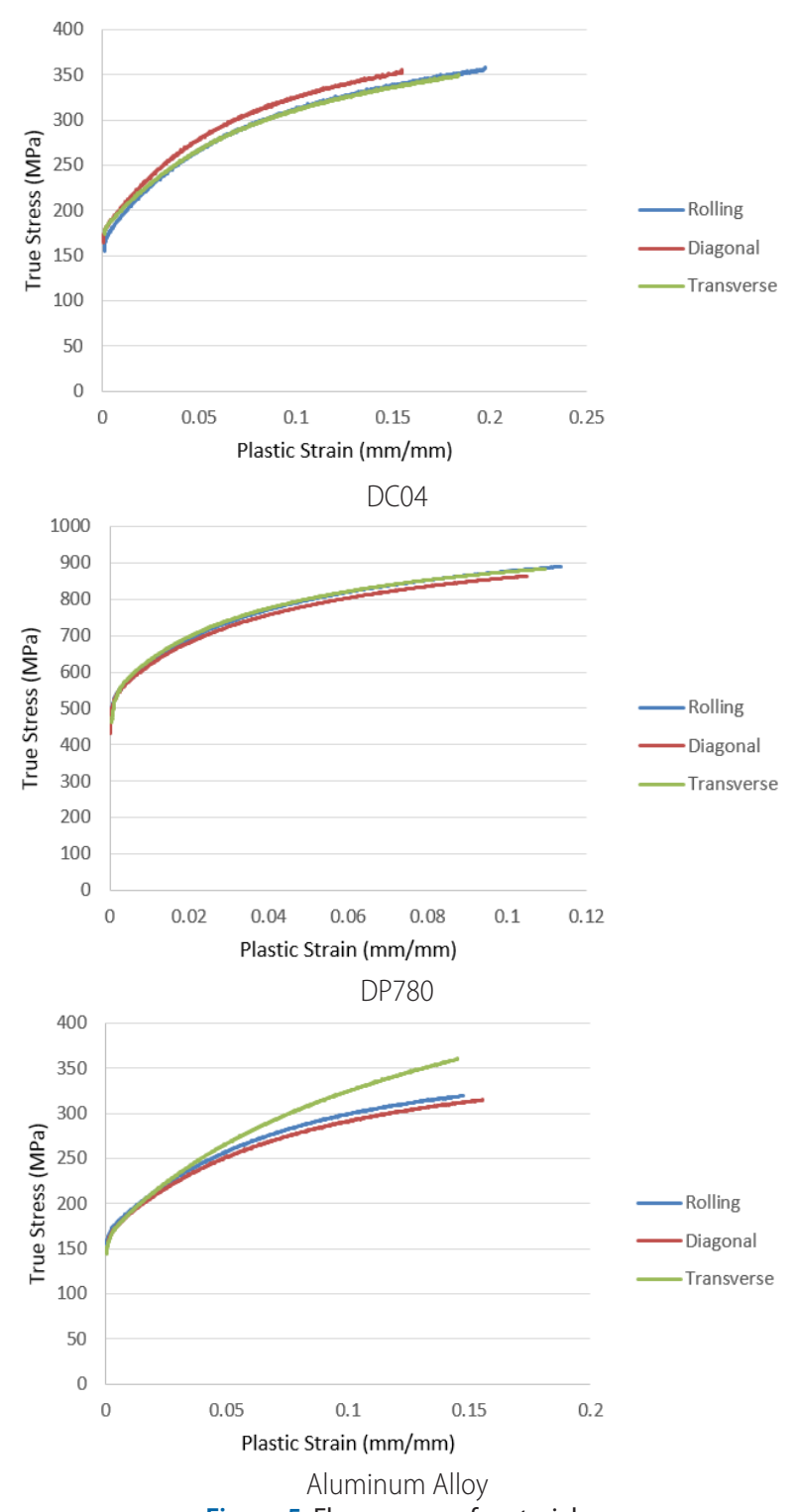

Figure 5. Flow curves of materials

Flow stress, tensile stress, and elasticity modulus of materials are calculated using engineering stress-strain curves however strength coefficient $(\mathrm{K})$ and hardening exponent $(\mathrm{n})$ are calculated using flow curves. These mechanical properties are used as input parameters for plasticity models. Some plasticity models in this study admit material as anisotropic so anisotropy coefficients must be calculated for materials. For this purpose, a second tensile test set is performed to calculate anisotropy coefficients. In these tests, three different lines are added between gauge length to measure sample width and thickness. In literature, the optimum measurement stage is admitted as an $18-20 \%$ elongation step [19] so all measurements are performed at $20 \%$ elongation. In this step, test machine is stopped and width-thickness measurements are obtained by a caliper. By using these measurements width and thickness strains are calculated to obtain anisotropy coefficients of materials. Anisotropy coefficients are calculated for all lines between gauge length then the average of the results are admitted as material anisotropy coefficient. An example of a tensile test sample for determining anisotropy coefficient can be seen in Fig. 6 .
All performed tests for this study are evaluated and mechanical properties for all materials in all directions are summarized in Table 1.

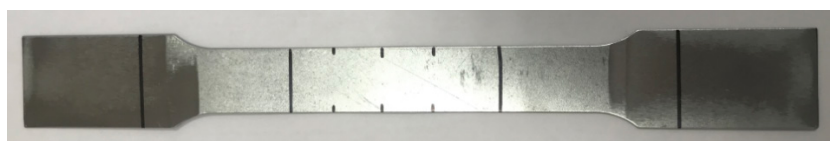

Figure 6. Test sample for determining anisotropy coefficient

Table 1. Mechanical properties of materials obtained from uniaxial tensile tests

\begin{tabular}{|c|c|c|c|c|c|c|c|}
\hline \multicolumn{2}{|c|}{} & $\begin{array}{c}\text { Elasticity } \\
\text { Modulus } \\
(\mathrm{GPa})\end{array}$ & $\begin{array}{c}\text { Yield } \\
\text { Stress } \\
(\mathrm{MPa})\end{array}$ & $\begin{array}{c}\text { Tensile } \\
\text { Stress } \\
(\mathrm{MPa})\end{array}$ & $\begin{array}{c}\mathrm{K} \\
(\mathrm{MPa})\end{array}$ & $\mathrm{n}$ & $\mathrm{r}$ \\
\hline \multirow{3}{*}{ DC04 } & $\mathrm{RD}$ & 170 & 154,88 & 293,30 & 484,28 & 0,19 & 1,92 \\
\cline { 2 - 9 } & $45^{\circ}$ & 180 & 163,72 & 304,07 & 486,63 & 0,18 & 1,35 \\
\cline { 2 - 8 } & $90^{\circ}$ & 185 & 173,78 & 291,06 & 467,41 & 0,18 & 2,21 \\
\hline \multirow{3}{*}{ DP780 } & $\mathrm{RD}$ & 210 & 457,83 & 791,36 & 1150,27 & 0,12 & 0,71 \\
\cline { 2 - 9 } & $45^{\circ}$ & 200 & 428,96 & 774,90 & 1096,98 & 0,11 & 0,88 \\
\cline { 2 - 9 } & $90^{\circ}$ & 212 & 460,59 & 789,23 & 1175,44 & 0,13 & 0,83 \\
\hline \multirow{2}{*}{$\begin{array}{c}\text { Alumi- } \\
\text { num }\end{array}$ Alloy } & $\mathrm{RD}$ & 76,5 & 154,27 & 275,20 & 433,31 & 0,17 & 0,77 \\
\cline { 2 - 8 } & $45^{\circ}$ & 74.2 & 138,62 & 268,90 & 432,22 & 0,18 & 0,76 \\
\cline { 2 - 8 } & $90^{\circ}$ & 76 & 143,19 & 270,43 & 510,27 & 0,21 & 0,87 \\
\hline
\end{tabular}

\subsection{Plasticity Modeling}

In this section, the plasticity models used in this study are presented. Within the context of this study, von Mises, Hill48, Hill-93, Barlat-89, and Hu-2003 models are studied. Yield loci and yield stresses - anisotropy coefficients in different directions are obtained with the plasticity models for all materials and compared with the experimental results for evaluating plasticity models.

\subsection{1 von Mises Criterion}

This criterion is presented in 1933 by von Mises [20], and this criterion known as maximum shape distortion energy criterion too. The general form of the von Mises criterion can be written as in Eq. (1).

$$
\frac{1}{6}\left[\left(\sigma_{11}-\sigma_{22}\right)^{2}+\left(\sigma_{22}-\sigma_{33}\right)^{2}+\left(\sigma_{33}-\sigma_{11}\right)^{2}\right]+\sigma_{12}^{2}+\sigma_{23}^{2}+\sigma_{31}^{2}=\frac{\sigma_{3}^{2}}{3}
$$

Sheet metal materials are used within the scope of the study, and all plasticity models used as plane stress forms $\left(\sigma_{3}=0\right.$, $\sigma_{13}=0, \sigma_{23}=0$ ) since sheet metal forming processes known as plane stress problems. Plane stress form of the von Mises criterion can be seen in Eq. (2).

$$
2 f\left(\sigma_{i j}\right)=F\left(\sigma_{22}-\sigma_{33}\right)^{2}+G\left(\sigma_{33}-\sigma_{11}\right)^{2}+H\left(\sigma_{11}-\sigma_{22}\right)^{2}+2 L \sigma_{23}^{2}+2 M \sigma_{31}^{2}+2 N \sigma_{12}^{2}=1
$$

This criterion admits isotropic material and isotropic hardening rule.

\subsubsection{Hill-48 Criterion}

R. Hill [21] is presented an anisotropic yield criterion in 1948. In this model, the material has an anisotropy at three orthogonal symmetry planes also this criterion admits isotropic hardening behavior. General form of Hill-48 criterion can be written as in Eq. (3).

$$
\begin{aligned}
& 2 f\left(\sigma_{i j}\right)=F\left(\sigma_{22}-\sigma_{33}\right)^{2}+G\left(\sigma_{33}-\sigma_{11}\right)^{2}+H\left(\sigma_{11}-\sigma_{22}\right)^{2} \\
& +2 L \sigma_{23}^{2}+2 M \sigma_{31}^{2}+2 N \sigma_{12}^{2}=1
\end{aligned}
$$

where F, G, H, L, M, and N constants depend on anisot- 
ropy coefficients. Plane stress form of the criterion is given in Eq.(4).

$2 f\left(\sigma_{i j}\right)=F\left(\sigma_{22}-\sigma_{33}\right)^{2}+G\left(\sigma_{33}-\sigma_{11}\right)^{2}+H\left(\sigma_{11}-\sigma_{22}\right)^{2}+2 L \sigma_{23}^{2}+2 M \sigma_{31}^{2}+2 N \sigma_{12}^{2}=1$

Relation of F, G, H, and N constants with anisotropy coefficients can be written in Eq. (5).

$$
\begin{aligned}
& F=\frac{r_{0}}{\left(1+r_{0}\right)\left(r_{90}\right)} \\
& G=\frac{1}{\left(1+r_{0}\right)} \\
& H=\frac{r_{0}}{\left(1+r_{0}\right)} \\
& N=\frac{\left(r_{0}+r_{90}\right)\left(2 r_{45}+1\right)}{\left.\left(2\left(1+r_{0}\right)\right) r_{90}\right)}
\end{aligned}
$$

where $r_{0}, r_{45}$, and $r_{90}$ represents anisotropy coefficients of rolling, diagonal, and transverse directions. This criterion has an important advantage since the model has a simple assumption of defining material anisotropy, and this criterion is still one the most used model for material analyses.

\subsubsection{Hill-93 Criterion}

In 1993, R. Hill improved the plastic behavior model for anisotropic materials under complex loads applied trough planar orthotropic axes [22]. This model is presented for materials (especially like aluminum and brass) which have approximately equal yield stresses but different anisotropy coefficients in rolling and transverse directions. This situation is known as "anomalous behavior of second order". Hill-93 model can be written as Eq. (6).

$$
\frac{\sigma_{1}^{2}}{\sigma_{0}^{2}}-\frac{c \sigma_{1} \sigma_{2}}{\sigma_{0} \sigma_{90}}+\frac{\sigma_{2}^{2}}{\sigma_{90}^{2}}+\left\{(p+q)-\frac{\left(p \sigma_{1}+q \sigma_{2}\right.}{\sigma_{b}}\right\} \frac{\sigma_{1} \sigma_{2}}{\sigma_{0} \sigma_{90}}=1
$$

Here, c, p, and q coefficients depend on yield stress and anisotropy coefficients in different directions. "c" coefficient can be written as Eq. (7).

$$
\frac{c}{\sigma_{0} \sigma_{90}}=\frac{1}{\sigma_{0}^{2}}+\frac{1}{\sigma_{90}^{2}}-\frac{1}{\sigma_{b}^{2}}
$$

p and q coefficients are given in Eq. (8) and Eq. (9), respectively.

$$
\begin{aligned}
& p=\left[\frac{2 r_{0}\left(\sigma_{b}-\sigma_{90}\right)}{\left(1+r_{0}\right) \sigma_{0}^{2}}-\frac{2 r_{90} \sigma_{b}}{\left(1+r_{90}\right) \sigma_{90}^{2}}+\frac{c}{\sigma_{0}}\right] \frac{1}{\frac{1}{\sigma_{0}}+\frac{1}{\sigma_{90}}-\frac{1}{\sigma_{b}}} ; \\
& q=\left[\frac{2 r_{90}\left(\sigma_{b}-\sigma_{90}\right)}{\left(1+r_{90}\right) \sigma_{90}^{2}}-\frac{2 r_{0} \sigma_{b}}{\left(1+r_{0}\right) \sigma_{0}^{2}}+\frac{c}{\sigma_{90}}\right] \frac{1}{\frac{1}{\sigma_{0}}+\frac{1}{\sigma_{90}}-\frac{1}{\sigma_{b}}} ;
\end{aligned}
$$

This criterion needs 5 material parameters for defining yield function $\left(\mathrm{r}_{0}, \mathrm{r}_{90}, \sigma_{0}, \sigma_{90}, \sigma_{\mathrm{b}}\right)$. Here, $\sigma_{\mathrm{b}}$ can be defined as yield stress value in the hydraulic bulge test. All of these material parameters can be obtained from tensile tests with two directions (rolling and transverse), and a hydraulic bulge test.

\subsubsection{Barlat-89 Criterion}

In 1989, Barlat and Lian presented a criterion for materials with planar anisotropy [23]. Barlat-89 criterion can be writ- ten as Eq. (10).

$$
f=a\left|k_{1}+k_{2}\right|^{M}+a\left|k_{1}-k_{2}\right|^{M}+c\left|2 k_{2}\right|^{M}=2 \sigma_{e}^{M}
$$

Here " $M$ " exponent depends on the crystal structure of materials. k1 and k2 coefficients can be written as Eq. (11).

$$
k_{1}=\frac{\sigma_{11}+h \sigma_{22}}{2} ; k_{2}=\left[\left(\frac{\sigma_{11}-h \sigma_{22}}{2}\right)^{2}+p^{2} \sigma_{12}^{2}\right]^{1 / 2}
$$

a, c, and h represents material constants and can be written as Eq. (12).

$$
h=\sqrt{\left(\frac{r_{0}}{1+r_{0}}\right)\left(\frac{1+r_{90}}{r_{90}}\right)}
$$

"p" parameter can be found by optimization. Barlat- 89 model is one of the most used models in finite element analyses since the model has a simple construction and needs a few number of material parameters.

\subsubsection{Hu-2003 Criterion}

In 2003, Hu presented a new plasticity model by improving Hill-48 criterion [24]. The general form of $\mathrm{Hu}-2003$ criterion can be written as Eq. (13).

$$
\begin{aligned}
& f(\bar{\sigma})=\frac{1}{\sigma_{0}^{4}} \sigma_{1}^{4}-\frac{4 r_{0}}{\left(1+r_{0}\right) \sigma_{0}^{4}} \sigma_{1}^{3} \sigma_{2} \\
& +\left(\frac{1}{\sigma_{b}^{4}}-\frac{1}{\sigma_{0}^{4}}-\frac{1}{\sigma_{90}^{4}}+\frac{4 r_{0}}{\left(1+r_{0}\right) \sigma_{0}^{4}}+\frac{4 r_{90}}{\left(1+r_{90}\right) \sigma_{90}^{4}}\right) \sigma_{1}^{2} \sigma_{2}^{2}-\frac{4 r_{90}}{\left(1+r_{90}\right) \sigma_{90}^{4}} \sigma_{1} \sigma_{2}^{3}+ \\
& \frac{1}{\sigma_{90}^{4}} \sigma_{y}^{4}+\left(\frac{16}{\left(1+r_{45}\right) \sigma_{45}^{4}}-\frac{2}{\sigma_{b}^{4}}\right)\left(\sigma_{1}^{2}+\sigma_{2}^{2}-\sigma_{1} \sigma_{2}\right) \sigma_{12}^{2} \\
& +\left(\frac{1}{\sigma_{b}^{4}}+\frac{16 r_{45}}{\left(1+r_{45}\right) \sigma_{45}^{4}}\right) \sigma_{12}^{4}=1
\end{aligned}
$$

$\mathrm{Hu}-2003$ criterion can model the plastic behavior of a material using 7 parameters as yield stresses and anisotropy coefficents in rolling, diagonal, and transverse directions and yield stress of hydraulic bulge test.

\section{VALIDATION OF PLASTICITY MODELS}

In this stage of the study, yield loci of plasticity models are obtained firstly. Yield locus can be defined as two-dimensional boundary limits of material yield for plane stress problems and must be closed, smooth, and convex. Inside of the yield locus represents the elastic area of the material. A sample yield locus schematic and experimental data on locus are shown in Fig. 7. In this study, yield loci of DC04, DP780, and aluminum alloy are obtained by five different plasticity model. Prediction performance of plasticity models is evaluated by investigating the positions of experimental yield stress values on yield loci. Evaluation of yield loci obtained using plasticity models is shown in Fig. 8-10. In the second stage of the study, angular variations of the yield stress ratio and anisotropy coefficients are predicted by plasticity models between rolling direction $\left(0^{\circ}\right)$ to transverse direction $\left(90^{\circ}\right)$. Prediction results are compared with experimental values in three directions. Comparison results are given in Fig. 11-13. 


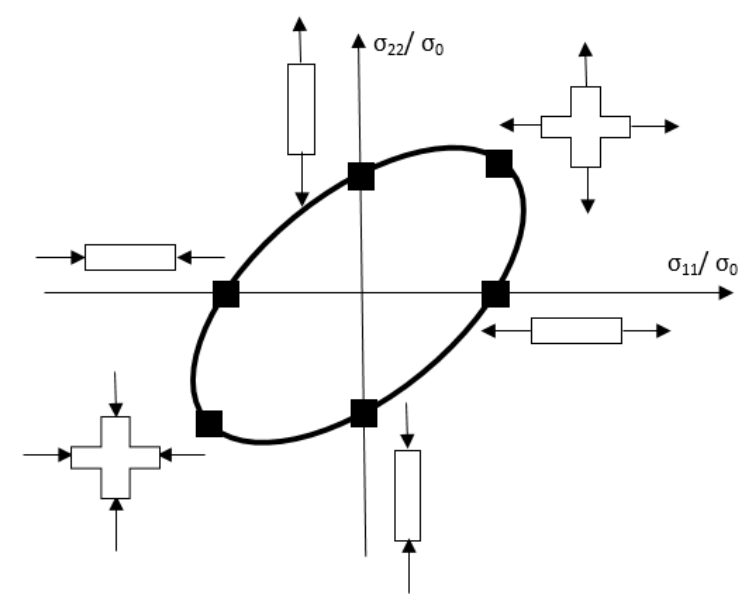

Figure 7. A sample yield locus and material yield points with different experiments on locus

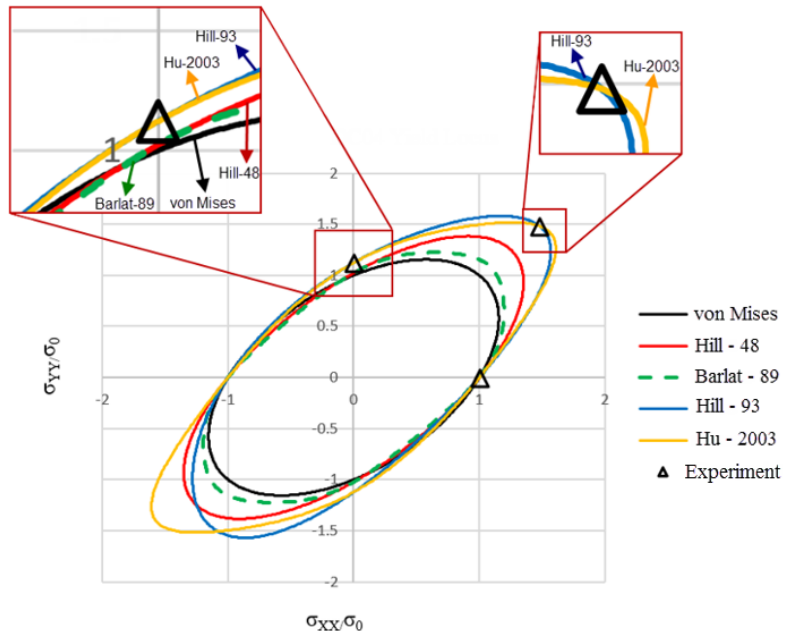

Figure 8. Yield loci of plasticity models for DC04

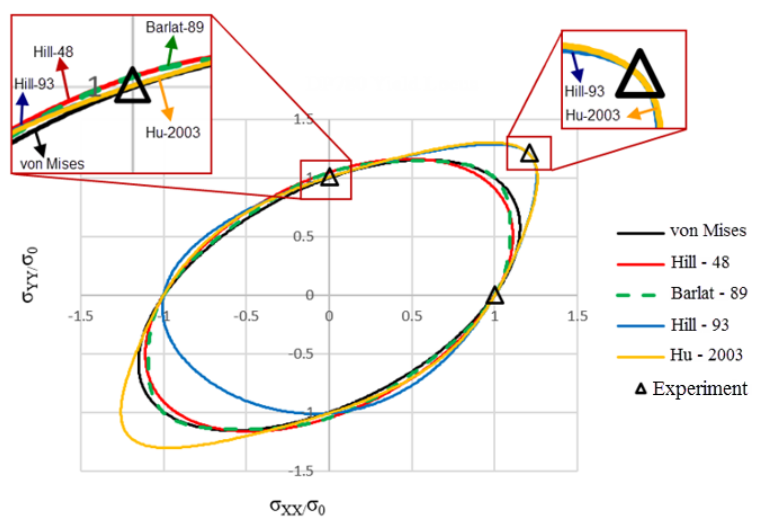

Figure 9. Yield loci of plasticity models for DP780

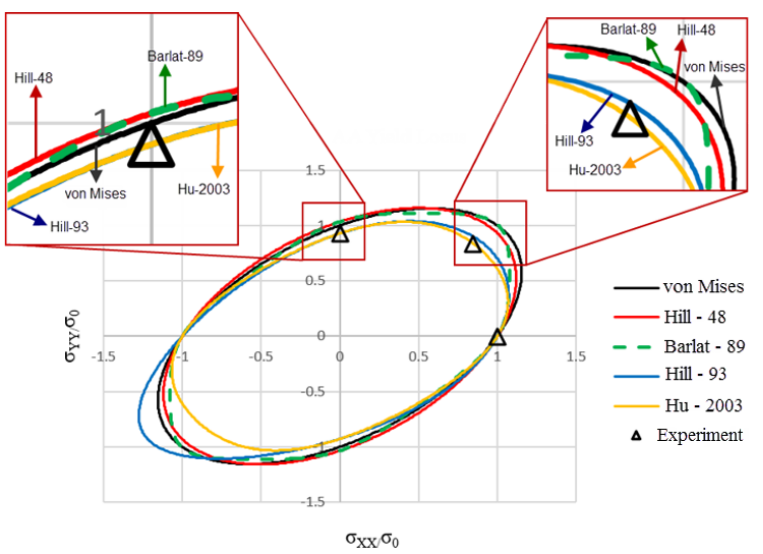

Figure 10. Yield loci of plasticity models for Aluminum alloy
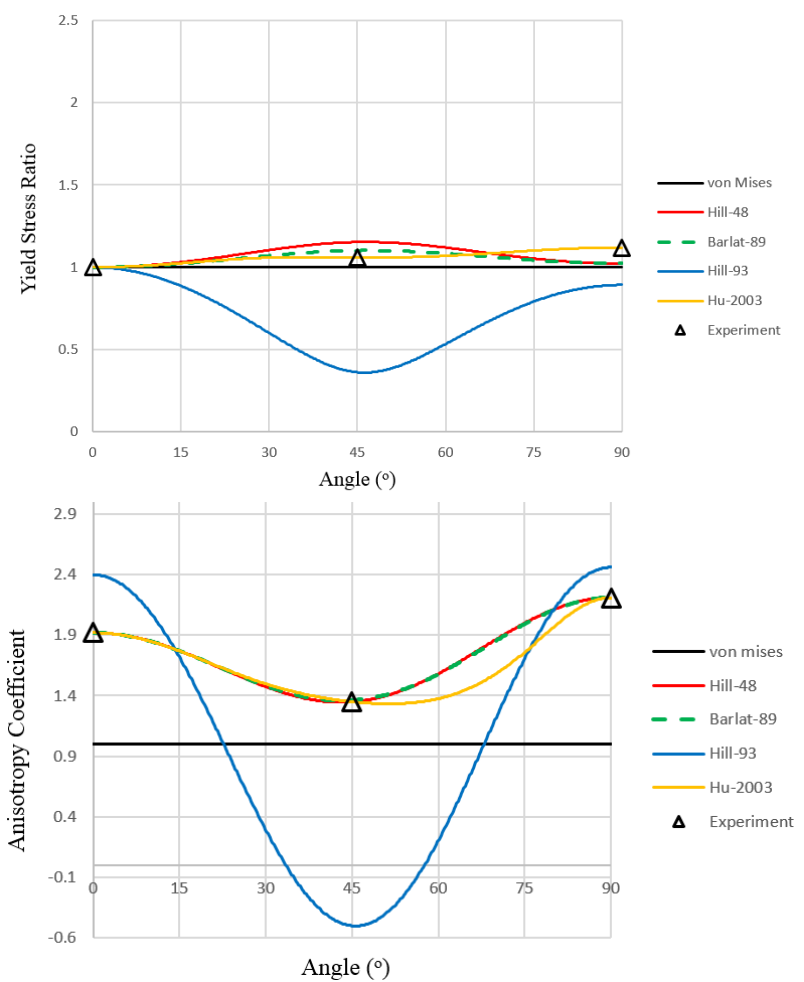

Figure 11. Comparison of plasticity model predictons for DC04
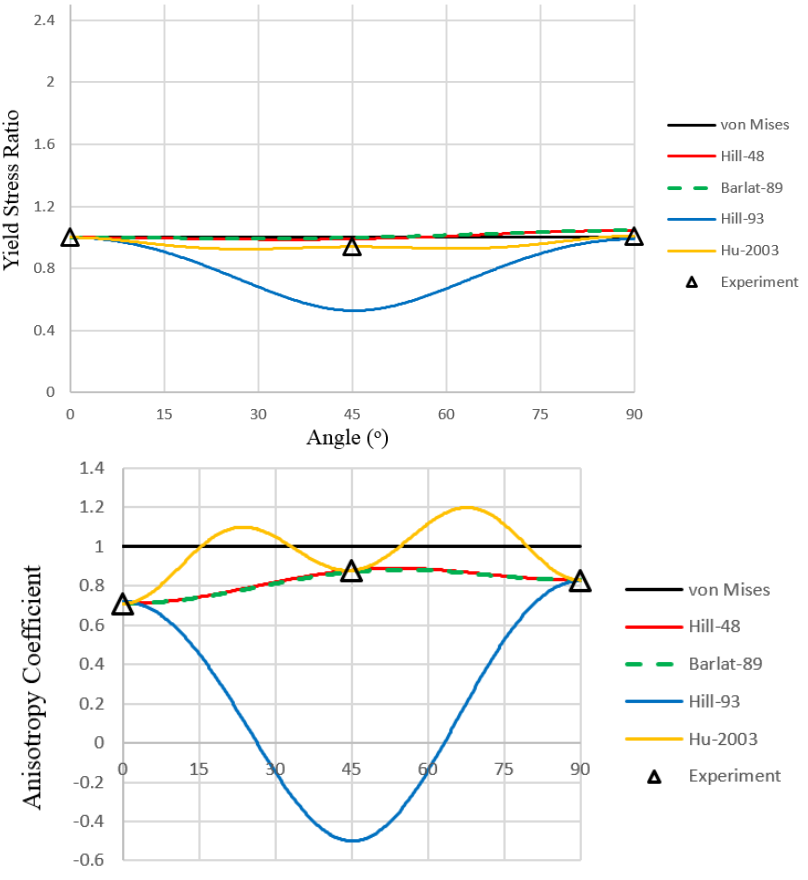

Angle $\left(^{\circ}\right)$

Figure 12. Comparison of plasticity model predictons for DP780

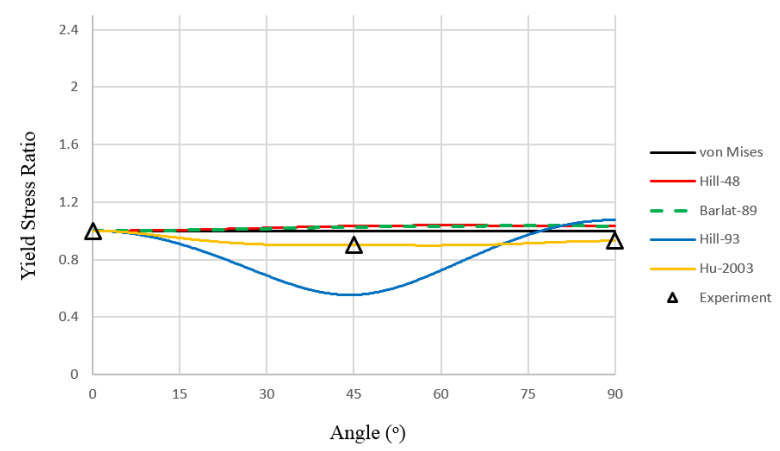




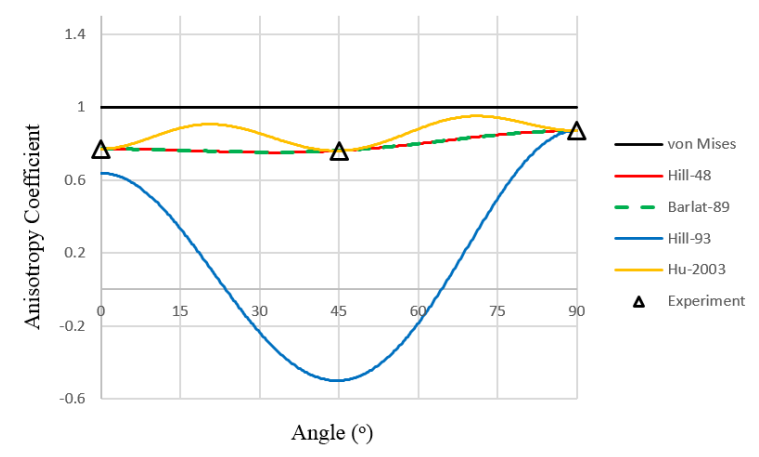

Figure 13. Comparison of plasticity model predictons for Aluminum alloy

For presenting prediction performance of plasticity models clearer, experimental results for rolling direction, diagonal direction, and transverse direction are compared with predictions of plasticity models. Comparison results are given in Fig. 14-16.

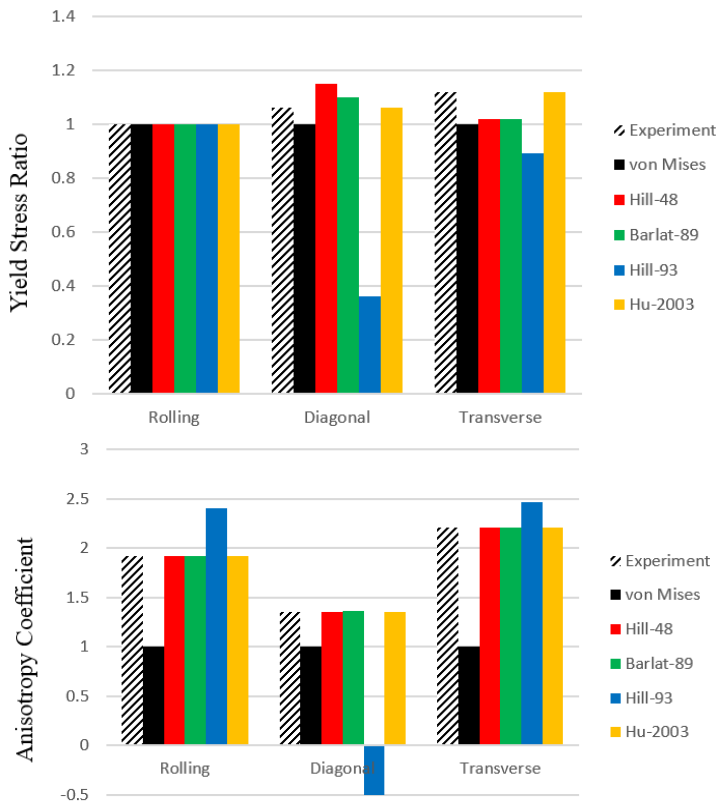

Figure 14. Comparison of plasticity model predictons for DC04

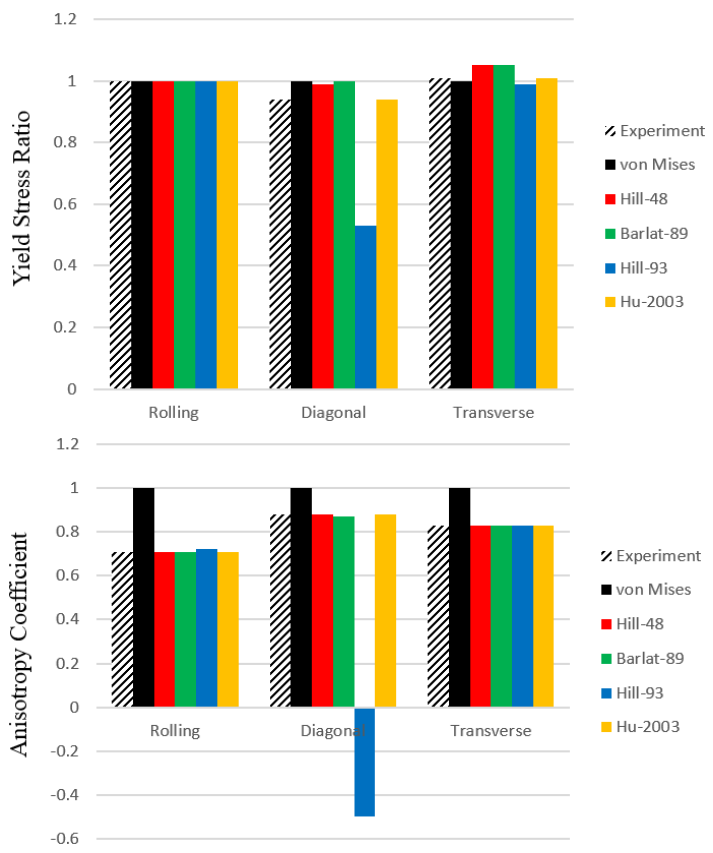

Figure 15. Comparison of plasticity model predictons for DP780

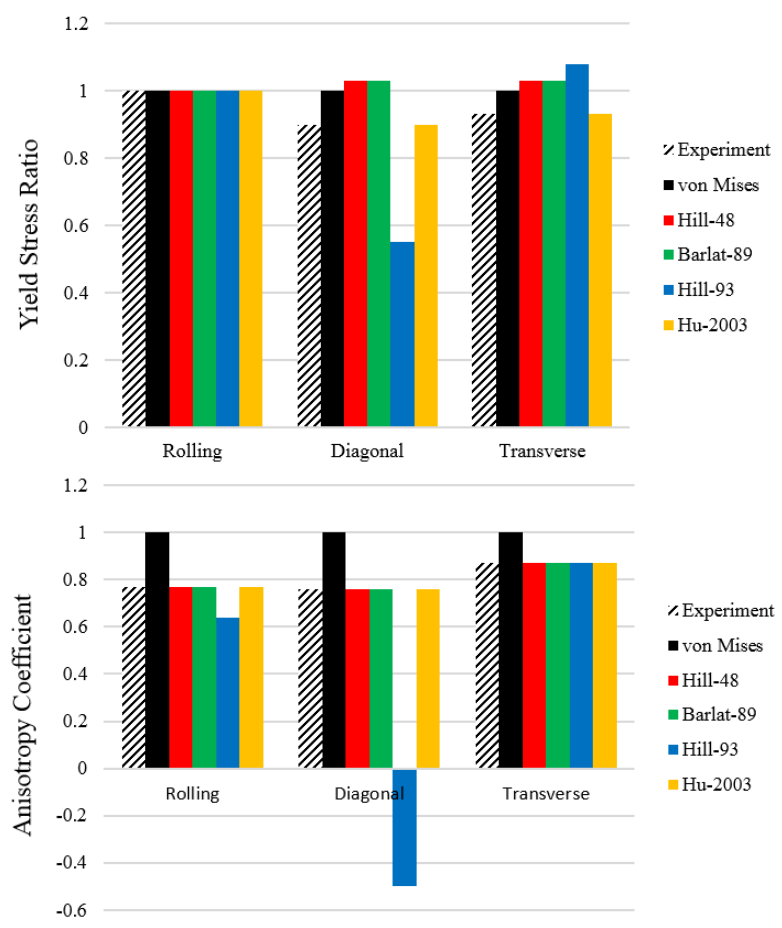

Figure 16. Comparison of plasticity model predictons for aluminum alloy

\section{CONCLUSIONS}

In this study, it is aimed to evaluate the prediction performance of plasticity models, which are used in finite element analyses, for different materials. For this purpose, prediction performance of plasticity models is evaluated using uniaxial tensile tests, and DC04, DP780, 6000 series aluminum alloy are used as materials.

In the first stage of the study, uniaxial tensile tests are performed for three directions (rolling, diagonal, transverse), and mechanical properties of materials are obtained. Besides, anisotropy coefficients of materials are determined by another uniaxial tensile test set for all directions. In this data set, mechanical properties are used as input parameters for plasticity models besides, anisotropy coefficients and yield stress values in three directions are used as validation parameters. In the second stage of the study plasticity modeling for all materials is performed using von Mises, Hill-48, Hill93, Barlat-89, and Hu-2003 plasticity models utilizing yield loci, and directional variations of yield stress and anisotropy coefficients. Plasticity model predictions are evaluated by comparing with experimental data.

As a result, it is seen that the isotropic von Mises plasticity model has a poor prediction capacity for all materials. If an evaluation is made between anisotropic criteria, Hill-93 and Hu-2003 models have a good biaxial stress prediction in yield locus. However, the Hill-93 criterion has a very poor prediction capacity for all materials through directional yield stress and anisotropy coefficient predictions of these models. In this sense, Hu-2003 criterion has an accurate prediction capacity for both yield locus and directional estimations. This situation can be explained as there is not an input for diagonal direction in the Hill-93 criterion hence this criterion have a poor performance for diagonal predic- 
tions. When it comes to Hill-48 and Barlat- 89 criteria these two models are unable to define biaxial stresses in yield locus. However, rolling and transverse stresses predictions have a very good agreement with experimental data in yield locus, and directional anisotropy coefficient and yield stress estimations seem effective for planar variations. Ultimately, the $\mathrm{Hu}-2003$ criterion becomes prominent for all materials. This result is due to the number of input parameters of the Hu-2003 model. This model performs by 7 parameters as yield stresses and anisotropy coefficients in rolling, diagonal, transverse directions, and biaxial yield stress. These parameters can be obtained by uniaxial tensile tests for three directions (rolling, diagonal, transverse) and a hydraulic bulge test. In this sense, $\mathrm{Hu}-2003$ criterion distinguishes with a high prediction capacity in despite of using simple and less number of mechanical tests.

\section{RERERENCES}

[1] Yoon, J. W., Dick, R. E., \& Barlat, F. (2011). A new analytical theory for earing generated from anisotropic plasticity. International Journal of Plasticity, 27(8), 1165-1184.

[2] Kuroda, M., \& Tvergaard, V. (2000). Forming limit diagrams for anisotropic metal sheets with different yield criteria. International Journal of Solids and Structures, 37(37), 5037-5059.

[3] Schmidt, I. (2005). Some comments on formulations of anisotropic plasticity. Computational materials science, 32(3-4), 518-523.

[4] Firat, M., Kaftanoglu, B., \& Eser, O. (2008). Sheet metal forming analyses with an emphasis on the springback deformation. journal of materials processing technology, 196(1-3), 135-148.

[5] Köleoğlu Gürsoy, Ö, \& Esener, E. (2019). Malzeme Modellerinin Sac Metal Sonlu Elemanlar Analizi Tahmin Performansına Etkisinin Değerlendirilmesi. Bilecik Şeyh Edebali Üniversitesi Fen Bilimleri Dergisi, 6(1).

[6] Li, X., Yang, Y., Wang, Y., Bao, J., \& Li, S. (2002). Effect of the material-hardening mode on the springback simulation accuracy of $V$-free bending. Journal of Materials Processing Technology, 123(2), 209-211.

[7] Banabic, D., Comsa, D. S., Sester, M., Selig, M., Kubli, W., Mattiasson, K., \& Sigvant, M. (2008, September). Influence of constitutive equations on the accuracy of prediction in sheet metal forming simulation. In Numisheet (pp. 37-42).

[8] Mars, J., Wali, M., Jarraya, A., Dammak, F., \& Dhiab, A. (2015). Finite element implementation of an orthotropic plasticity model for sheet metal in low velocity impact simulations. Thin-Walled Structures, 89, 93-100.

[9] Kuwabara, T., Hashimoto, K., lizuka, E., \& Yoon, J. W. (2011). Effect of anisotropic yield functions on the accuracy of hole expansion simulations. Journal of Materials Processing Technology, 211(3), 475-481.

[10] Roters, F., Eisenlohr, P., Hantcherli, L., Tjahjanto, D. D., Bieler, T. R., \& Raabe, D. (2010). Overview of constitutive laws, kinematics, homogenization and multiscale methods in crystal plasticity finite-element modeling: Theory, experiments, applications. Acta Materialia, 58(4), 1152-1211.

[11] Ozsoy, M., Esener, E., Ercan, S., \& Firat, M. (2014). Springback predictions of a dual-phase steel considering elasticity evolution in stamping process. Arabian Journal for Science and Engineering, 39(4), 3199-3207.
[12] Javanmardi, M. R., \& Maheri, M. R. (2019). Extended finite element method and anisotropic damage plasticity for modelling crack propagation in concrete. Finite Elements in Analysis and Design, 165, $1-20$.

[13] Zhou, R., Roy, A., \& Silberschmidt, V. V. (2019). A crystal-plasticity model of extruded AM30 magnesium alloy. Computational Materials Science, 170, 109140.

[14] Meng, L., Chen, W., Yan, Y., Kitamura, T., \& Feng, M. (2019). Modelling of creep and plasticity deformation considering creep damage and kinematic hardening. Engineering Fracture Mechanics, 218, 106582.

[15] Feng, D. C., Ren, X. D., \& Li, J. (2018). Cyclic behavior modeling of reinforced concrete shear walls based on softened damage-plasticity model. Engineering Structures, 166, 363-375.

[16] Esmaeilpour, R., Kim, H., Park, T., Pourboghrat, F., Xu, Z., Mohammed, B., \& Abu-Farha, F. (2018). Calibration of Barlat Yld2004-18P yield function using CPFEM and 3D RVE for the simulation of single point incremental forming (SPIF) of 7075-O aluminum sheet. International Journal of Mechanical Sciences, 145, 24-41.

[17] Soare, S. C., \& Barlat, F. (2011). A study of the Yld2004 yield function and one extension in polynomial form: A new implementation algorithm, modeling range, and earing predictions for aluminum alloy sheets. European Journal of Mechanics-A/Solids, 30(6), 807-819.

[18] Standard, A. S. T. M. (2011). E8/E8M. Standard test methods for tension testing of metallic materials, 3, 66 .

[19] Zang, S. L., Thuillier, S., Le Port, A., \& Manach, P. Y. (2011). Prediction of anisotropy and hardening for metallic sheets in tension, simple shear and biaxial tension. International Journal of Mechanical Sciences, 53(5), 338-347.

[20] Mises R (1913) Mechanics of solids in plastic state. Göttinger Nachrichten Mathematical Physics 4:582-592

[21] Hill, R. (1948). A theory of the yielding and plastic flow of anisotropic metals. Proceedings of the Royal Society of London. Series A. Mathematical and Physical Sciences, 193(1033), 281-297.

[22] Hill, R. (1993). A user-friendly theory of orthotropic plasticity in sheet metals. International Journal of Mechanical Sciences, 35(1), 19-25.

[23] Barlat, F., \& Lian, K. (1989). Plastic behavior and stretchability of sheet metals. Part I: A yield function for orthotropic sheets under plane stress conditions. International journal of plasticity, 5(1), 51-66.

[24] Hu, W. (2003). Characterized behaviors and corresponding yield criterion of anisotropic sheet metals. Materials Science and Engineering: A, 345(1-2), 139-144. 